\title{
Emerging Issues Regarding the Employment and Career Development of Americans with Disabilities
}

\author{
Fong Chan ${ }^{\mathrm{a}, *}$ and Phillip Rumrill ${ }^{\mathrm{b}}$ \\ ${ }^{\mathrm{a}}$ University of Wisconsin-Madison, Madison, WI, USA \\ ${ }^{\mathrm{b}}$ Kent State University, Kent, OH, USA
}

Despite encouraging trends in Federal legislation and a growing societal commitment to the ideals of equal opportunity and social justice, poverty and income inequality continue to have dire consequences for Americans with disabilities (Murali \& Oyebode, 2004; World Health Organization, 1995). There is ample evidence to indicate that income inequality produces psychosocial stress, and high levels of stress lead to deteriorating health and higher mortality (Dutta, Gervey, Chan, Chou, \& Ditchman, 2008; Krause, Carter, Pickelsimer, \& Wilson, 2008; Murali \& Oyebode, 2004). In the United States, the labor force participation rate of $19.4 \%$ for people with disabilities is significantly lower than the $69.4 \%$ rate for people without disabilities (U.S. Department of Labor, 2014). There is no doubt that the lack of employment opportunities excludes people with disabilities from full community inclusion and participation, stalls upward mobility, and greatly affects their health-related quality of life (U.S. Senate

*Address for correspondence: Fong Chan, Ph.D., CRC, APA Fellow, Norman L. and Barbara M. Berven Professor of Rehabilitation Psychology, Chair, Department of Rehabilitation Psychology and Special Education, University of Wisconsin-Madison, 401 Education Building, 1000 Bascom Mall, Madison, WI 53706, USA. E-mail: chan@education.wisc.edu.
Committee on Health, Education, Labor and Pensions [SCHELP], 2012).

Participation in competitive integrated employment is considered a fundamental human right and crucial to the physical and psychological well-being of people with disabilities (Dutta et al., 2008). Senator Tom Harkin, in his report on making employment of people with disabilities a national priority, indicated (SCHELP, 2012) that the jobless rates of individuals with disabilities remain unacceptably high. Senator Harkin underscored the importance of helping people with disabilities obtain and retain competitive integrated employment that can lead to quality careers, economic security, and the opportunity to join the middle class. As a result, throughout the recently passed Workforce Innovation and Opportunity Act (WIOA; U.S. Department of Education, 2014), especially in the amendments to the Rehabilitation Act, there is a significant emphasis on local labor market analysis, employer engagement, customized training, postsecondary education, pre-employment transition services, youth with disabilities, interagency collaboration, and accountability.

Clearly, rehabilitation professionals are expected to develop new skills and competencies and utilize innovative demand-side employment strategies and 
evidence-based practices in vocational rehabilitation to improve employment outcomes and career pathways for people with disabilities. With that in mind, this special issue of the Journal of Vocational Rehabilitation was developed to present a wide range of subject matter and research methods that bear on the present and future employment of people with disabilities. Contributors to this special issue have examined such phenomena as the quality of employment outcomes for people with disabilities, workplace discrimination, the relationship between age and disability, assistive technology, strategies for leveraging community stakeholders to provide employment opportunities for people with disabilities, and the employment concerns and outcomes of specific disability populations. Readers will note a variety of research designs and methods in the articles presented in this issue, including parametric and non-parametric analyses of large national data sets such as the U.S. Equal Employment Opportunity Commission's Integrated Mission System and the Rehabilitation Services Administration's 911 Case Report data, qualitative analysis, organizational case studies, multiple regression analysis, and multi-level analysis.

In preparing this issue, we invited nine vocational rehabilitation research groups to share their original research related to contemporary practices that are helpful for the implementation of the mandates of the WIOA and the accompanying amendments to the Rehabilitation Act. The first two articles address employer engagement. Dutta and her colleagues (2016) reported the findings of their efforts to conduct World Café focused community conversations to identify service needs of transition age youth with disabilities. Using a general inductive approach to analyze their community conversations data, areas of service for transition age youth with disabilities, they found five service areas that are important to transition age youth with disabilities; they are (a) demand side focused vocational rehabilitation services, (b) transition services for building employment skills, (c) connecting existing community based resources, (d) advocacy services, and (e) parent and family involvement in transition. Phillips et al. (2016) conducted interviews with staff and managers associated with the $n$ Ablement job placement division of SPR Consulting, a medium-sized information technology company in Chicago. Using the consensual qualitative research (CQR) approach to analyze the qualitative data, findings of their study revealed unique advantages and challenges for people with disabilities receiving in-house, demandside employment and training services. Results of Phillips et al.'s study also suggest multiple opportunities for information technology companies that include a focus on disability in their workforce diversity and inclusion programs.

The next two articles examined discrimination experiences of people with multiple sclerosis and middle-aged and younger adults with disabilities in the workplace. Roessler and his colleagues (2016) analyzed data from the Integrated Mission System of the U.S. Equal Employment Opportunity Commission (EEOC) to investigate workplace discrimination experiences of people with multiple sclerosis across three phases of Americans with Disabilities Act (ADA) implementation: Phase I (pre-Sutton decision era, 1992-1999), Phase II (Sutton decision era, 2000-2008), and Phase III (ADA Amendments Act era, 2009-2011). They found that, over the three periods of time, more typically disenfranchised individuals (e.g., women and minorities) sought ADA protections, possibly due to publicity, support from advocates, and membership in a culture supporting diversity and nondiscrimination. $\mathrm{Li}$ and her colleagues (2016) analyzed the same EEOC data to evaluate the discrimination experiences of middleage charging parties with disabilities (ages 35-54 years) in comparison to younger adult charging parties with disabilities (ages 25-34 years). They found that compared to younger adult charging parties, middle-age charging parties were more likely to be male and Caucasian; more likely to file allegations on the basis of diabetes, heart/cardiovascular disease, back impairments, and cancer; and less likely to file allegations on the basis of anxiety disorders, manic depressive disorder, schizophrenia, or other psychological impairments.

The next three articles analyzed the U.S. Department of Education, Rehabilitation Services Administration Case Service Report (RSA-911) data to examine person-environment factors and rehabilitation service variables' influence on employment outcomes of people with disabilities receiving services from state vocational rehabilitation agencies. Chan and his colleagues (2016) used RSA-911 data along with state-level economic indicators and vocational rehabilitation agency characteristics data to identify predictors of employment quality for people with disabilities using multilevel analysis. They found that individual characteristics were stronger predictors of employment quality than environmental factors and rehabilitation service variables. Personal 
characteristics also explained a considerable amount of between-state differences in quality employment outcomes. For example, improvement in educational attainment was related to higher employment quality, which is consistent with the emphasis of the WIOA on postsecondary education as a pathway to good jobs with benefits. Conversely, the proportion of consumers receiving social security benefits in a particular state and state per capita income factors were strong environmental predictors of employment quality. Kaya and his colleagues (2016) used a purposeful selection multivariate logistic regression analysis to investigate the extent to which demographic characteristics, Social Security disability benefits, and vocational rehabilitation services influence competitive employment outcomes for transition-age youth with autism spectrum disorders. They identified on-the-job support, job placement services, rehabilitation technology, occupational/vocational training, other services, job search assistance, vocational counseling and guidance, and job readiness training as rehabilitation services significantly associated with successful employment outcomes. In addition, higher levels of educational attainment, receiving a greater number of VR services, and not receiving Social Security disability benefits were positively associated with competitive employment outcomes. Huang and her colleagues (2016) investigated the individual characteristics of consumers receiving assistive technology services in state vocational rehabilitation agencies. They reported that older adults, individuals with sensory impairments and physical impairments, persons with higher educational attainment, and people who were employed at application had higher propensities to receive assistive technology services than did other consumers.

The last two articles focus on positive psychology as postsecondary education and vocational rehabilitation interventions. Rumrill and his colleagues (2016) described the use of cognitive support technology as a positive psychology intervention for postsecondary students with traumatic brain injuries (TBI). Through the use of cognitive support technology in the form of iPads and a set of iPad applications, they were able to help students with TBI meet their support needs and increase their personal, social, academic, and vocational capacities as they pursue their academic and career goals. Finally, Umucu and his associates (2016) evaluated two positive psychology constructs (secure attachment and self-efficacy) for their relationships to employment status among people with spinal cord injuries. The findings of their study indicate significant bivariate relationships among secure attachment, self-efficacy, and employment status. Self-efficacy was found to be a significant mediator of the relationship between secure attachment and employment status. This study provides preliminary support for positive psychology as a conceptual framework for vocational rehabilitation (VR) practice with people with disabilities, which has implications for development and implementation of alternative or complementary VR interventions.

Taken together, the articles contained in this special issue reflect the breadth and depth of the methodological approaches that rehabilitation researchers use to gain a thorough understanding of the employment concerns and outcomes of Americans with disabilities in the present WIOA and Americans with Disabilities Act Amendments Act era. They also focus on disability populations whose needs for employment-related supports may not be well understood (e.g., transitionage youth; middle-aged workers; college students; and people with autism, multiple sclerosis, traumatic brain injuries, and spinal cord injuries). It is our hope that this issue presents content that will aid vocational rehabilitation professionals in developing evidencebased practices to promote employment opportunities and long-term career success for people with disabilities.

We wish to thank the authors who contributed articles to this issue. Our gratitude also goes to Journal of Vocational Rehabilitation's Editor, Dr. Paul Wehman, for the opportunity to complete this work.

\section{References}

Chan, F., Wang, C. C., Fitzgerald, S., Muller, V., Ditchman, N., \& Menz, F. (2016). Personal, environmental, and service-delivery determinants of employment quality for state vocational rehabilitation consumers: A multilevel analysis. Journal of Vocational Rehabilitation, 45(1), 5-18.

Dutta, A., Gervey, R., Chan, F., Chou, C. C, \& Ditchman, N. (2008). Vocational rehabilitation services and employment outcomes of people with disabilities: A United States study. Journal of Occupation Rehabilitation, 18, 326-334.

Dutta, A., Kundu, M., Johnson, E., Chan, F., Trainor, A., Blake, R., \& Kristy, R. (2016). Community conversations: Engaging stakeholders to improve employment-related transition services for youth with emotional and behavioral disabilities. Journal of Vocational Rehabilitation, 45(1), 43-51.

Huang, I. C., Cheing, G., Rumrill, P., Bengtson, K., Chan, F., Telzlaff, J., \& Snitker, M. (2016). Characteristics of people with disabilities receiving assistive technology services in vocational rehabilitation: A logistic regression analysis. Journal of Vocational Rehabilitation, 45(1), 63-72. 
Kaya, C., Chan, F., Rumrill, P., Hartman, E., Wehman, P., Iwanaga, K., Pai, C.-H., \& Avellone, L. (2016). Vocational rehabilitation services and competitive employment for transition-age youth with autism spectrum disorders. Journal of Vocational Rehabilitation, 45(1), 73-83.

Krause, J. S., Carter, R. Pickelsimer, E., \& Wilson, D. (2008). A prospective study of health and risk of mortality after spinal cord injury. Archives of Physical Medicine and Rehabilitation, 89, 1482-1491.

Murali, V., \& Oyebode, F. (2004). Poverty, social inequality and mental health. Advances in Psychiatric Treatment, 10, 216224.

Phillips, B., Morrison, B., Deiches, J., Yan, M. C., Strauser, D., Chan, F., \& Kang, H. J. (2016). Employer-driven disability services provided by a medium-sized information technology company: A qualitative case study, Journal of Vocational Rehabilitation, 45(1), 85-96.

Rossesler, R. T., Rumrill, P. D., Li, J., \& McMahon, B. T. (2016). The workplace discrimination experiences of people with multiple sclerosis across three phases of Americans with Disabilities Act implementation. Journal of Vocational Rehabilitation, 45(1), 27-41.

Rumrill, P., Elias, E., Hendricks, D. J., Jacob, K., Leopold, A., Nardone, A.,. . .McMahon, B. T. (2016). Promoting cognitive support technology use and employment success among postsecondary students with traumatic brain injuries. Journal of Vocational Rehabilitation, 45(1), 53-61.

Umucu, E., Lee, B., Wu, J.-R., Chan, F., Blake, J., Brook, J., \& Catalano, D. (2016). Self-efficacy as a mediator for the relationship between secure attachment style and employment status in individuals with spinal cord injuries. Journal of Vocational Rehabilitation, 45(1), 97-106.

U.S. Department of Labor (2014a). Office of Disability Employment Policy-Current disability employment statistics. Retrieved from http://www.dol.gov/odep/

U.S. Department of Labor (2014b). Earnings and unemployment rates by educational attainment. Retrieved from http:// data.bls.gov/cgi-bin/print.pl/emp/ep_chart_001.htm

U.S. Senate Committee on Health, Education, Labor and Pensions (2012). Unfinished business: Making hiring people with disabilities a national priority. Washington, DC: Author. Retrieved from http://www.harkin.senate.gov/documents/pdf/ 500469b49b364.pdf.

World Health Organization (WHO). (1995). Bridging the gaps. Geneva, Switzerland: Author. 